\title{
Involucramiento del Padre en la Crianza: Una Mirada Triádica de las Relaciones Familiares Tempranas ${ }^{1}$
}

\author{
Paternal Involvement in Child Rearing: \\ A Triadic View of Early Family Interactions
}

\author{
Francisca Pérez Cortés ${ }^{2}$ \\ Universidad de Chile, Santiago, Chile \\ Marcia Olhaberry \\ Pontificia Universidad Católica de Chile, Santiago, Chile \\ (Rec: junio 2013 - Acep: febrero 2014)
}

\begin{abstract}
Resumen
Considerando los beneficios de una paternidad activa en el desarrollo de niños y niñas, así como en las dinámicas familiares en general, los profesionales del área de la salud deben ser agentes activos en la promoción de la participación de madres y padres en la crianza. El siguiente artículo busca introducir en el contexto nacional una herramienta que amplía la mirada del desarrollo infantil y las relaciones al interior de la familia, desde la díada a la tríada. El Lausanne Trilogue Play (LTP) es una herramienta observacional, desarrollada por FivazDepeursinge, Frascarolo y Corboz-Warnery (1996) que permite la evaluación estandarizada de la "alianza familiar", corroborando la importancia de incorporar al padre en los estudios de procesos e interacciones familiares, así como la existencia de un efecto familiar tríadico desde etapas tempranas.
\end{abstract}

Palabras clave: paternidad activa, desarrollo infantil, coparentalidad, alianzas familiares.

\begin{abstract}
Considering the benefits that an active paternity has in child development and in general family dynamics, health professionals should be active agents in promoting maternal and paternal care. The following article seeks to introduce in the national context an instrument that contributes in enlarging the perspective in child development and family relationships, from the dyad to the triad. The Lausanne Trilogue Play (LTP) is an observational tool, developed by Fivaz-Depeursinge, Frascarolo y Corboz-Warnery (1996) that allows a standardized evaluation from the "family alliance", corroborating the importance of incorporating the father in the studies of family process and interactions, as well as the existence of a triadic family effect since early stages.
\end{abstract}

Keywords: active paternity, child development, coparentality, family alliances

\footnotetext{
1 Este estudio recibió financiamiento de la Comisión Nacional de Investigación Científica y Tecnológica, CONICYT Chile, al Proyecto Fondecyt de Postdoctorado º3120109 y al Núcleo Milenio NS10008 Intervención Psicológica y Cambio en Depresión, financiado por el Ministerio de Economía, Fomento y Turismo, del Gobierno de Chile.

2 Correspondencia dirigida a: Francisca Pérez. Universidad de Chile, Facultad de Medicina. Av. Salvador 486, comuna de Providencia, Santiago de Chile. Tel: (56-2) 227488 55. E-mail: fsperezcortes@ug.uchile.cl.
} 


\section{Introducción}

La familia es la unidad relacional básica a través de la cual se mantienen y transmiten ciertas rutinas y prácticas, como por ejemplo las funciones y roles que observamos en hombres y mujeres, las cuales no son producto del resultados natural de su sexo, sino de un sistema de género (DeBarbieri, 1992). El modelo predominante que asumía el hombre en la cultura occidental moderna estaba ligado a su función proveedora y protectora y su rol principal estaba en torno al trabajo, la vida pública, la provisión de bienes para el hogar y la autoridad familiar. Por su parte, el rol femenino se vinculaba con la vida privada, el cuidado del hogar, la crianza de los hijos/as y la entrega de apoyo afectivo, entre otros (Valdés \& Olavarría, 1998).

Los complejos cambios culturales y económicos, ocurridos a partir de la inclusión de los métodos de control de natalidad y del incremento de la participación femenina en la fuerza laboral, han generado un cambio en la constitución familiar así como en la distribución de los roles de género tradicionales. Este nuevo escenario ha propiciado la igualdad de derechos entre hombres y mujeres, transformando la crianza de los hijos/as en una tarea compartida entre los miembros de la pareja. Actualmente un gran número de parejas forman familias nucleares con mujeres que trabajan fuera del hogar, lo que ha generado expectativas sociales respecto a la participación de los padres en la crianza de sus hijos (Pleck \& Pleck, 1997).

En países occidentales desarrollados los padres se han involucrado más en la crianza durante las últimas décadas, sin embargo, aún dedican menos tiempo a esta actividad que las madres, a pesar de que éstas trabajen fuera del hogar (Coltrane \& Adams, 2001; Hofferth, Pleck, Stueve, Bianchi, \& Sayer, 2002; Pleck \& Masciadrelli, 2004). Pleck (1985) mostró que el empleo de la madre fuera del hogar no aumentaba de forma significativa el nivel de involucramiento del padre en la crianza. En la misma línea, Lamb, Pleck, Charnov y Levine (1987) mostraron que el empleo materno aumenta el compromiso y disponibilidad del padre hacia sus hijos/as, pero tiene un pequeño impacto en el grado de responsabilidad paterna en la crianza de éstos. En parejas donde ambos trabajan fuera del hogar la diferencia en el tiempo que madres y padres dedican a sus hijos es significativa, las madres pasan alrededor de 2.5 horas más de tiempo con sus hijos/as que los padres en un día de trabajo (Feldman, 2000).

No obstante los avances y cambios en las configuraciones de roles familiares, aún la mayor carga en términos de tiempo y responsabilidad de las tareas domésticas, de cuidado y de crianza sigue estando en manos de las mujeres. En Chile, si bien los hombres están cada vez más interesados en participar en la paternidad, todavía su desempeño está focalizado en roles como el juego, los paseos y el apoyo en tareas escolares (Aguayo, Correa, \& Cristi, 2011). De este modo, aún estamos lejos de lograr la corresponsabilidad en las tareas parentales y domésticas entre hombres y mujeres (Valdés \& Olavarría, 1998).

Muchas veces, las instituciones públicas y privadas encargadas de salud y educación reproducen los estereotipos de género dominantes, considerando a los hombres actores secundarios o poco relevantes en las tareas de crianza. Los profesionales de la salud, por ejemplo, al no incluir a los padres en las entrevistas (controles prenatales, controles de salud niño/a sano, etc.) y al no considerarlos en las indicaciones y cuidado de los niños/as limitan la posibilidad de avanzar hacia la corresponsabilidad en la crianza, reforzando la idea de que el cuidado de los niños/as es una función principalmente materna. Sin embargo, resultados de la Encuesta IMAGES (Aguayo et al., 2011), refieren que nueve de cada diez hombres sí tiene interés en participar junto a sus hijos/as en los diferentes momentos de interacción con el sistema de salud y educativo. La encuesta señala también que existen barreras culturales y materiales que dificultan una mayor participación de los hombres, tales como horarios de trabajo, permisos laborales, y creencias tradicionales que consideran como exclusivos de las mujeres los temas de salud, de cuidado o educativos, etc.

$\mathrm{Al}$ considerar la evidencia que sostiene que la presencia activa de los padres impacta positivamente en el desarrollo psicosocial de sus hijos/as (Allen \& Daly, 2007; Sarkadi, Kristiansson, Oberklaid, \& Bremberg, 2008) y que la coparentalidad aporta soporte psicológico y emocional vital para el crecimiento y desarrollo de todos los integrantes de la familia, se hace necesario un cambio en las miradas y prácticas en relación a los roles de género tradicionales en la vida familiar. Es así como se vuelve fundamental dejar de considerar al padre como un actor secundario en la crianza de sus hijos/as, y facilitar una incorporación y participación activa de éste.

El siguiente artículo busca dar a conocer en el medio nacional una herramienta de evaluación e intervención de las interacciones familiares que involucra a ambos padres como actores activos en el proceso de socialización de sus hijos/as. Bajo la premisa que considera la participación de cada individuo y las relaciones 
recíprocas entre ellos como elementos centrales en la emergencia de un estilo familiar armónico y cohesivo que apoya un desarrollo óptimo en el niño/a (Belsky, 1981; Cowan \& Cowan, 2002; McHale \& Cowan, 1996). El Lausanne Trilogue Play (Fivaz-Depeursinge, Frascarolo, \& Cornoz-Warnery, 1996) permite la evaluación de las relaciones familiares tríadicas, "madre, padre e hijo/a", considerando al padre como un protagonista más dentro de la dinámica familiar.

\section{Contribuciones de la incorporación del padre en la comprensión del desarrollo y la salud mental infantil}

Históricamente las investigaciones en psicología del desarrollo, interacciones y procesos familiares se han centrado en la díada madre-hijo/a (Flykt, Kanninen, Sinkkonen, \& Punamäki, 2010; Teti, Gelfland, Messinger, \& Isabella, 1995; Weinberg \& Tronick, 1998). Es bien sabido que la presencia de sintomatología depresiva y estrés en las madres disminuye sus niveles de reciprocidad, sincronía y coordinación con su hijo/a, comprometiéndose la capacidad diádica y tríadica que permiten la regulación afectiva (Feldman, 2007; Rodrigo, Martín, Cabrera, \& Máiquez, 2009).

Si bien la mayoría de las investigaciones en infancia temprana se han centrado en el vínculo entre el bebé y su madre, un número creciente de estudios exploran la importancia del padre. Los primeros estudios que incluyeron al padre mostraron que su presencia tiene un impacto en las conductas de la madre y del hijo/a $y$ en la calidad del intercambio emocional entre ambos; influencia que sugiere un efecto familiar tríadico (Clarke-Stewart, 1978; Yogman, 1981). La evidencia muestra que los vínculos infantiles de apego se desarrollan hacia ambos progenitores y que los padres son capaces de proveer cuidados sensibles a sus hijos/as (Keller, 2007; Lamb, 1977; Lamb, 1982). Diversas investigaciones muestran que la presencia activa del padre impacta positivamente el desarrollo de los niños/ as en diversas áreas, como por ejemplo, un adecuado vínculo padre-hijo/a se asocia a ausencia de problemas conductuales en los niños/as (Verschueren \& Marcoen, 1999), así como a alta sociabilidad y a adecuado desarrollo cognitivo (Fagan \& Iglesias, 1999). Otros estudios señalan que la presencia del padre aumenta las probabilidades de que los hijos/as presenten una mejor salud mental en la adolescencia, disminuyendo además la sobrecarga de la madre en las tareas domésticas y de cuidado e incrementando su salud física y mental. Asimismo la presencia del padre suele aumentar el ingreso familiar, lo que impacta positivamente en la calidad de vida y en las posibilidades de desarrollo de los hijos/as (Allen \& Daly, 2007; Nock \& Einolf, 2008; Sarkadi et al., 2008).

En una revisión de 16 estudios longitudinales en los cuales se consideró la variable impacto de la presencia del padre, se encontró que quienes tuvieron un padre involucrado en su infancia presentaron en promedio menos problemas conductuales, menos conflictos con la ley, menor vulnerabilidad económica, mejor desarrollo cognitivo, mejor rendimiento escolar y menor estrés en la adultez (Sarkadi et al., 2008).

Por su parte, la ausencia del padre ha mostrado tener un impacto negativo en el desarrollo de los niños/ as, especialmente en el desarrollo de los niños varones (Dornes, 2006; Fthenakis, 1992). Además, interfiere además de manera negativa en el éxito terapéutico de una intervención centrada en el apego para tratar trastornos conductuales y de regulación y genera altos costos económicos y sociales directos e indirectos (Nock \& Einolf, 2008).

Las investigaciones en salud mental infantil y familiar muestran que la participación del niño/a en la tríada que integra junto a su madre y su padre, puede ayudar a resolver interacciones diádicas disfuncionales con uno de ellos, a partir de la intervención de un tercero que despliega un repertorio adecuado, sin coludirse ni excluir a ningún participante. Esta experiencia favorece además la regulación emocional en el niño/a durante la interacción, contribuyendo a la reducción de la tensión y el estrés (Fivaz-Depeursinge \& Favez, 2006). Otros autores han descrito otros beneficios para el niño/a, asociando la participación infantil en interacciones tríadicas con el padre y la madre a adecuada competencia social a los cuatro años (Hedenbro, 2006). En esta misma línea, estudios actuales describen que la adecuada salud mental del padre actúa como un factor protector para el niño/a en la relación entre la sintomatología depresiva materna e infantil (Gere et al., 2013).

Considerando la robusta investigación que destaca las contribuciones que realiza el padre en el desarrollo y la salud mental infantil, resulta de alta relevancia que clínicos e investigadores amplíen su mirada desde la díada a la tríada, incorporando en los diagnósticos y en el diseño de intervenciones tanto a la madre como al padre al momento de comprender las dificultades infantiles o promover salud mental. Esta nueva mirada implica considerar también la capacidad de ambos padres para trabajar juntos en las tareas y desafíos de la crianza. 


\section{Coparentalidad}

El nacimiento de un bebé genera cambios en la organización familiar, debiendo los padres crear condiciones que permitan atender las necesidades del nuevo integrante de la familia. Esta experiencia, modifica también la relación de pareja, de manera que frente a las dificultades y exigencias que este cambio genera, la expresión de afecto y complicidad actuarían como factores protectores para el adecuado ejercicio de la paternidad (Shapiro, Gottman, \& Carrere, 2000). Por otro lado, el apoyo emocional del padre hacia la madre, ha sido asociado a una adecuada sensibilidad materna frente a las señales del bebé, afectando positivamente la interacción diádica (Hyunjeong, Young-Joo, \& MiJa, 2006; Olhaberry \& Santelices, 2013; Valenzuela, 1997). En esta misma línea, estudios longitudinales en familia temprana muestran que los bebés que se desarrollan con el respaldo de padres que se apoyan mutuamente en su función parental, presentan mayor facilidad para participar en interacciones triangulares satisfactorias y responder de manera ajustada, que aquellos pertenecientes a familias con padres en conflicto (FivazDepeursinge \& Corboz-Warnery, 1999).

En este contexto, Minuchin (1995), destaca la importancia de la capacidad de los padres para coordinarse y ejercer una adecuada coparentalidad, que facilite la socialización y la crianza del bebé. La coperantalidad se refiere a la capacidad de compartir protagonismo, compromiso y apoyo mutuo en la crianza de los hijos/ as (McHale, 1995). Se considera positiva, cuando ambos padres se apoyan y solidarizan en sus esfuerzos por responder a las necesidades y comunicaciones de sus hijos/as (McHale \& Kuersten-Hogan, 2004). La armonía familiar permite a los niños/as el desarrollo de un sentimiento de bienestar y herramientas adaptativas que le ayudarán a insertarse sin tener que recurrir a defensas internalizantes o externalizantes. De esta forma, en un nivel tríadico, la calidad de la coparentalidad predice el ajuste social al ambiente preescolar (McHale \& Rasmussen, 1998), la apertura a los sentimientos negativos relativos a la separación (Favez et al., 2006), así como a modelos de apego más concordantes entre ambos padres (Caldera \& Lindsey, 2006). Por el contrario, una coparentalidad hostil y competitiva, así como discrepancias significativas entre los padres en el involucramiento o rechazo en la crianza durante la infancia temprana, pueden conducir a sentimientos de vacío y tristeza en el niño/a que se expresarán en sintomatología internalizante o externalizante durante la etapa preescolar (Elliston, McHale, Talbot, Parmley, \&
Kuersten-Hogan, 2008). Sin ir más lejos, el antagonismo en la coparentalidad y la falta de cohesión familiar observada durante la primera infancia o la etapa preescolar, se han asociado en estudios transversales y longitudinales a sintomatología clínica significativa en la escala Child Behavioral Checklist (Fivaz-Depeursinge et al., 1996), donde padres, cuidadores diarios y personal educativo reportan conductas agresivas y problemas internalizantes en los niños/as (Frosch \& Mangelsdorf, 2001; McHale \& Rasmussen, 1998), una probabilidad creciente de apegos inseguros (Frosch, Mangelsdorf, \& McHale, 2000) o no concordantes entre ambos padres (Caldera \& Lindsey, 2006), así como mayor agresión en la imaginería en el juego con muñecos que representan a la familia (McHale, Neugebauer, Radin, \& Schwartz, 1999).

Es así como una coparentalidad competitiva puede generar alianzas problemáticas al interior de la familia, mientras que una coparentalidad de apoyo genera alianzas cooperativas, las cuales pueden ser evaluadas a través del Lausanne Trilogue Play.

\section{El Lausanne Trilogue Play como método para evaluar las alianzas familiares}

El juego constituye una forma natural de relacionarse de los niños/as y a partir de ésta se expresan afectos, deseos y motivaciones. El juego entre padres e hijos/ as es una oportunidad para compartir estos afectos, desarrollar la reciprocidad, así como también es una instancia en que los niños/as aprenden a regular sus propios estados emocionales (Stern, 1985; Tronick, 1989).

Dado que en la sociedad occidental la mayoría de los niños/as nacen en familias biparentales y las interacciones desde el nacimiento son multipersonales más que estrictamente diádicas (Dunn, 1991), resulta esencial estudiar el juego en un nivel familiar. Sin embargo, son pocos los investigadores que han estudiado las relaciones tríadicas "madre-padre-hijo/a", principalmente por la falta de métodos apropiados, así como por su mayor complejidad. Mientras que en una díada hay sólo una forma posible de interacción, la investigación del juego tríadico resulta más compleja, ya que es necesario explorar cuatro tipos de configuraciones (tres díadas, una tríada), dado que cada integrante puede asumir un rol activo o tomar una posición pasiva.

El Lausanne Trilogue Play (LTP) es un instrumento observacional desarrollado por FivazDepeursinge, Frascarolo y Corboz-Warner (1996) que permite evaluar de forma estandarizada la interacción 
entre madre-padre e hijo/a. Actualmente existen tres versiones, el LTP prenatal (Corboz-Warnery \& Fivaz-Depeursinge, 2001), el LTP postnatal (FivazDepeursinge \& Corboz-Warnery, 1999) y el LTP para niños/as (toddler). El procedimiento consiste en que cada miembro de la familia se ubica en un asiento formando un triángulo que facilita el contacto visual y a través de una consigna la familia es alentada a jugar en cuatro fases: 1) padre o madre juegan activamente con el niño/a mientras el otro adulto sólo está presente, 2) luego estos roles se invierten entre los padres, 3 ) luego padre, madre y niño/a juegan activamente y 4) finalmente padre y madre interactúan y el niño/a juega solo. En el procedimiento prenatal las interacciones se realizan con un muñeco, en el postnatal la actividad se desarrolla sin juguetes y en el caso de los niños/as, se realiza alrededor de una mesa con un set de juguetes simples. Las interacciones se filman con dos cámaras sincronizadas, una enfocando a los padres y la otra al niño/a, luego se editan ambas grabaciones de manera tal de tener en un mismo cuadro a los tres integrantes de forma simultánea.
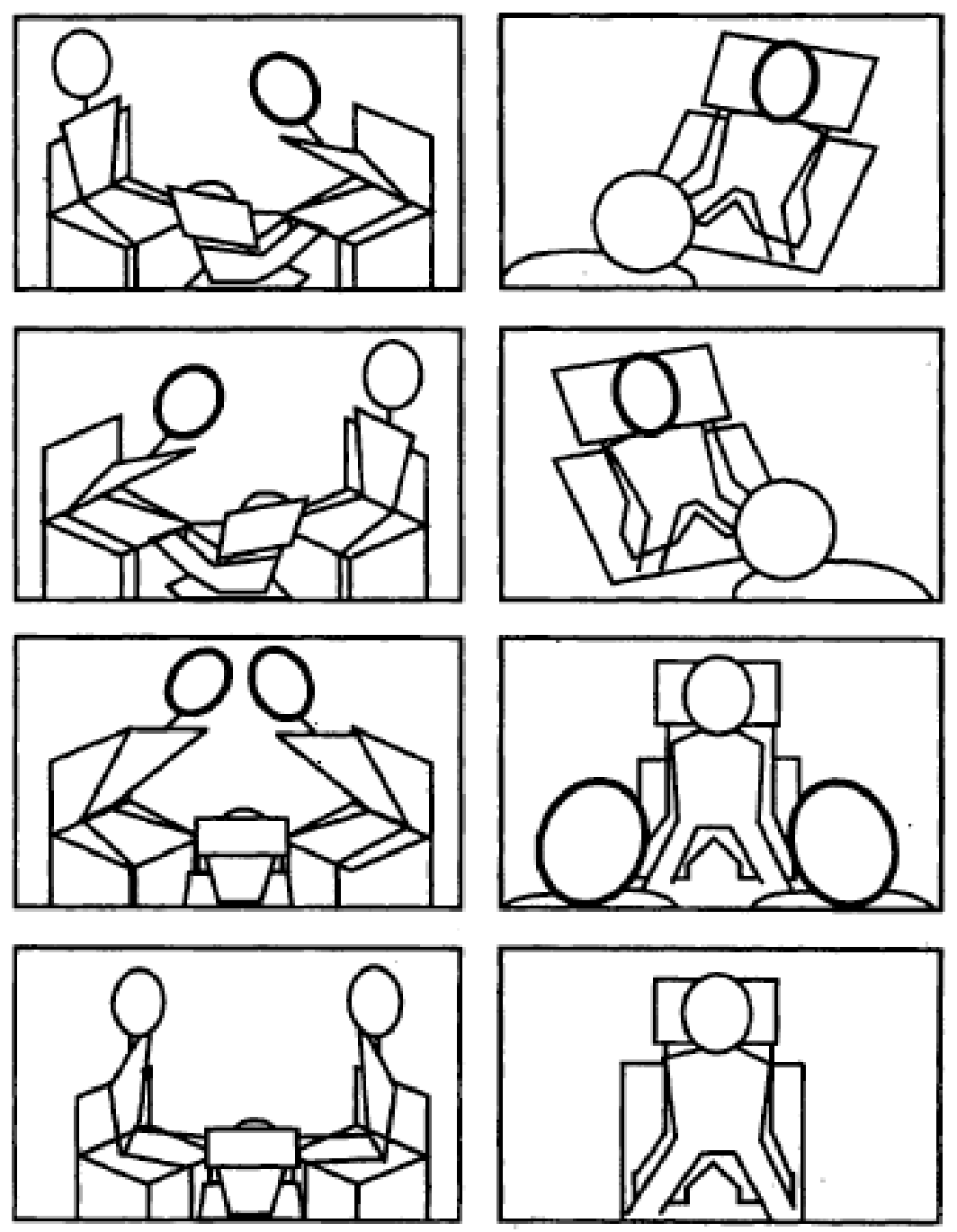

Figura 1.

Vista de las cuatro posiciones del LTP (versión postnatal) Fivaz- Depeursinge, Frascarolo, Corboz- Warnery (1996) 


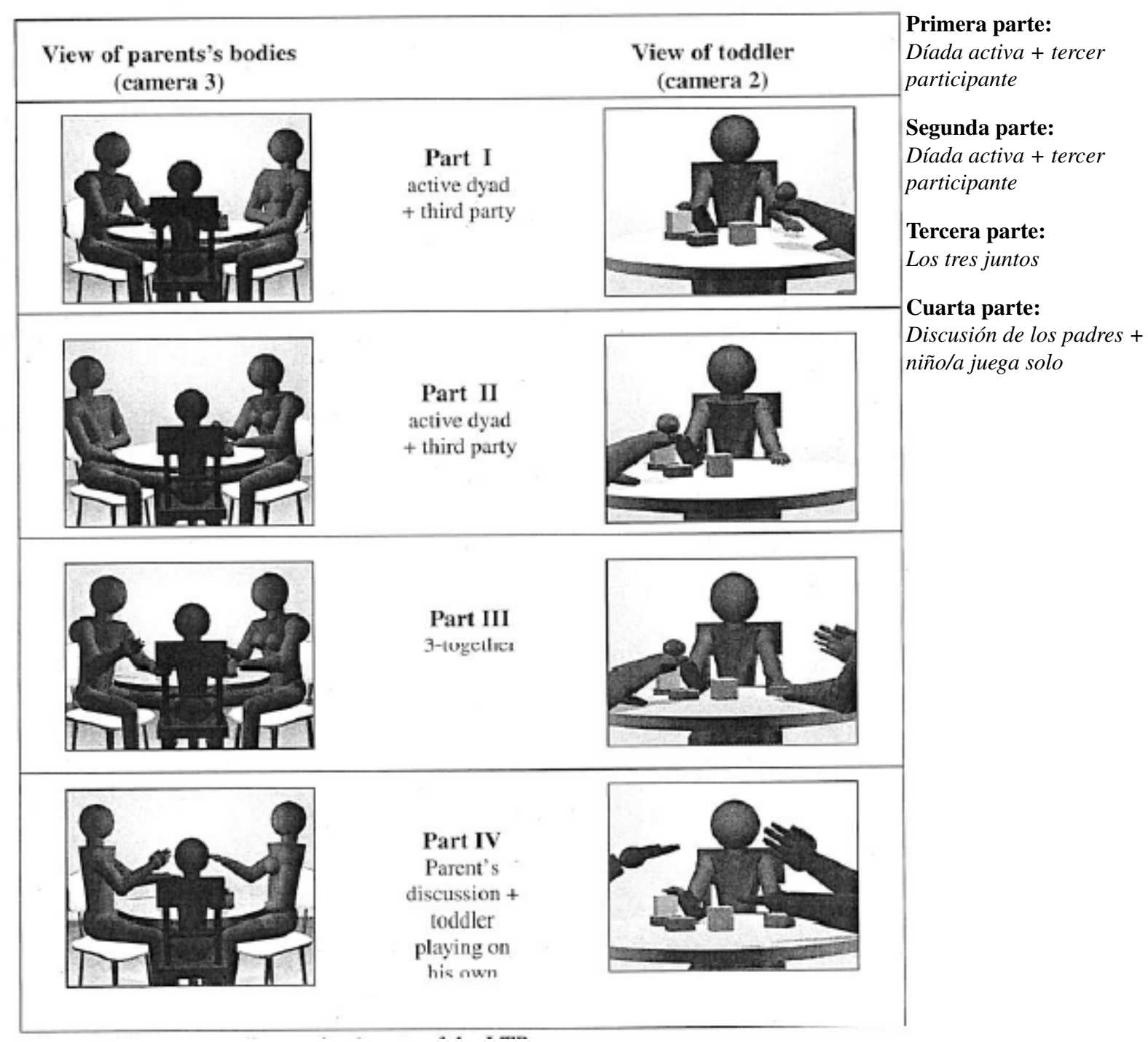

Figura 2.

Vista de las cuatro posiciones del LTP (versión infantil)

Centre d'Etude de la Famille (2007).

A través de microanálisis se analizan los cuatro tipos de configuraciones (tres díadas, una tríada), para lo cual se distinguen cuatro funciones que se dan en las interacciones, las cuales se encuentran imbricadas y siguen un orden jerárquico: 1) la participación (inclusión de los participantes), 2) la organización (los integrantes se mantienen en su rol), 3) la focalización (se comparte un foco común de atención) y 4) y el contacto afectivo (estar conectado). La primera función constituye la condición inicial necesaria, ya que en la configuración de la tríada todas las partes deben estar incluidas. $\mathrm{Si}$ una de las partes es excluída o se auto-excluye el juego se vuelve diádico o no existente. La segunda función hace referencia a que las partes mantengan su rol, ya sea el de participante activo u observador activo, sin que hayan interferencias. La tercera función hace referencia a la capacidad de compartir un foco común de atención y de co-construir un juego y por último, la cuarta función señala la capacidad de compartir afectos. $\mathrm{El}$ análisis de estas funciones en las configuraciones familiares a través del juego permite identificar la funcionalidad o disfuncionalidad de las interacciones familiares tríadicas, las cuales se pueden categorizar en "alianzas familiares", de acuerdo al grado de coordinación logrado por sus integrantes (Fivaz-Depeursinge \& Corboz-Warnery, 1999). 
La alianza cooperativa describe a familias cuyos miembros participan, logran coordinarse "suficientemente bien" en torno a una tarea conjunta, respetan sus roles, comparten un foco de atención e intercambian afectos positivos. El evaluador observa que las interacciones son coherentes y cohesionadas. La alianza conflictiva, por su parte, describe a familias cuyos miembros compiten, lo que se expresa a través de un conflicto que puede ser abierto o encubierto. Los padres no son capaces de coordinarse "suficientemente bien" para llevar a cabo la tarea, interrumpiéndose, provocando quiebres en la interacción y compitiendo por obtener la atención del niño/a. Si bien logran realizar juegos diádicos, les resulta más difícil realizar juegos tríadicos. Por último, la alianza desorganizada describe a familias que muestran interacciones que se caracterizan por la exclusión de uno de sus miembros y que a pesar de sus esfuerzos, no logran coordinación, cohesión ni contacto emocional entre sí.

Familias que presentan alianzas cooperativas tienden a fomentar el desarrollo socioemocional y autonomía de sus hijos/as, manteniendo límites claros y flexibles entre la díada parental y el niño/a. Por el contrario, es sabido que patrones familiares disfuncionales se asocian a resultados patológicos en los niños/as, como sintomatología psicosomática (FivazDepeursinge, Lopes, Python, \& Favez, 2009).

Lauretti y McHale (1997) observaron la responsividad parental durante interacciones díadicas y tríadicas, encontrando que en contextos en lo que hay tensión marital la sensibilidad de los padres hacia sus hijos/as declina significativamente. Es probable que en contextos donde exista tensión marital la atención de los padres esté más dirigida hacia su conflicto de pareja y por ende disminuiría la sensibilidad a las necesidades de sus hijos/as. De esta forma resulta evidente que la calidad de la coparentalidad tendrá un impacto en el desarrollo del niño/a (Belsky, Putnam, \& Crnic, 1996).

En el uso del LTP en contextos no clínicos se ha identificado que los bebés realizan ofertas relacionales a ambos padres, típicamente intercambiando a través de la mirada señales de placer o displacer entre ambos padres de forma equitativa (Lavanchy, 2002). En familias con alianzas funcionales estos intercambios son más frecuentes que en familias con alianzas disfuncionales. Asimismo el intercambio de afectos placenteros por sobre afectos displacenteros suele ser más frecuente en familias con alianzas funcionales que disfuncionales (Koller, 2004). En otras palabras, en familias con alianzas problemáticas los intercambios emocionales entre el bebé o niño/a y sus padres suelen ser más negativos. McHale, Fivaz-Depeursinge, Dickstein, Robertson y Daley (2008) replicaron estos resultados con una muestra de 110 bebés de tres meses de edad y además encontraron que existe una clara relación entre la capacidad de compartir afectos y la funcionalidad de la coparentalidad.

Por su parte, el uso del LTP en contextos clínicos ha mostrado que la capacidad tríadica es significativamente más baja en familias con antecedentes clínicos que familias no clínicas (Gertsch, Favez, Corboz-Warnery, \& Fivaz-Depeursinge, 1992). El LTP también ha sido utilizado como herramienta de intervención terapéutica con resultados favorables, utilizando el video feedback en familias con hijos con trastornos de personalidad (Viziello \& Nosadi, 2010).

\section{Discusión}

La modernidad ha generado nuevos desafíos a las familias, demandando una mayor participación en la crianza de los hijos/as por parte del padre y la madre. Si bien esto implica asumir roles que complejizan las relaciones de pareja y las tareas que cada uno debe realizar, estos cambios apuntan a la complementariedad y a la colaboración mutua, lo que redunda en mayores beneficios para el desarrollo y la salud mental infantil. La presencia del padre y la madre satisfechos con su relación de pareja y con una adecuada coparentalidad, enriquece las interacciones con los hijos/as y actúa al mismo tiempo como un elemento protector frente al riesgo de psicopatología infantil, regulando y corrigiendo mutuamente interacciones disfuncionales con el niño/a. El intercambio descrito brinda al niño/a experiencias distintas de sí mismo, de los otros significativos y sus las relaciones que enriquecen sus representaciones y favorecen su adaptación a la realidad.

En el presente artículo se presenta una nueva herramienta observacional, que evalúa las relaciones familiares tríadicas "madre, padre hijo/a" a través del juego. El Lausanne Trilogue Play ha sido usado tanto en investigación como en clínica, mostrando buenos índices de validez y confiabilidad. A través de un sistema de evaluación jerárquico, establece una serie de funciones que definen una interacción exitosa versus una problemática. La evaluación de estas cuatro funciones permite detectar diferencias individuales, las que se pueden abordar en una intervención terapéutica. Por ejemplo, si la función de participación no se logra, porque uno de los padres constantemente desvía su mirada de la interacción, entonces el niño/a no tendrá 
la posibilidad de experimentar la unión tríadica. La experiencia de conocerse a sí mismo/a junto a sus padres se verá entonces perjudicada, primando la experiencia de falta de disponibilidad de uno de los padres. Por su parte, familias que logran la participación de sus miembros, pero fallan en la organización de los roles (p.e. padres compiten por obtenera atención de sus hijos), los niños/as experimentarán un conflicto de lealtad, quedando atrapados entre sus padres. Cuando se logran las dos primeras funciones, pero se falla en la focalización, el niño/a no tendrá la experiencia de mantener la atención en juegos co-construidos. Finalmente, si se cumplen las cuatro funciones el niño/a no sólo tendrá la oportunidad de compartir la atención en un juego co-construido, sino que además tendrá la oportunidad de experimentar intersubjetividad, lo que constituye el objetivo fundamental del juego (Stern, 1985), y en este caso la intersubjetividad en un contexto tríadico (Fivaz-Depeursinge \& Corboz-Warnery, 1999).

De esta forma, evaluar qué funciones se cumplen y cuáles no da cuenta de los recursos así como los posibles riesgos dentro de la familia para el desarrollo del niño/a. Es así como la evaluación de las funciones provee un modelo útil de las interacciones familiares tanto para la investigación como para la clínica, ayudando a los clínicos a objetivar la evaluación intuitiva del funcionamiento familiar.

Como ya se mencionó, es posible distinguir tres tipos de alianzas familiares a partir del análisis de las interacciones tríadicas y el nivel de coordinación logrado en la actividad, las que pueden variar desde la más funcional a la más problemática. El tipo de alianza nos indica por un lado la necesidad o no de intervención psicoterapéutica para la familia, generando focos temáticos en los cuales se puede trabajar, y también permite realizar intervenciones preventivas en salud mental con niños/as pequeños/as o con padres gestantes que muestran dificultades. Este último punto resulta de alta relevancia ya que estudios longitudinales en alianzas familiares muestran que estas tienden a mantenerse estables durante los dos primeros años del niño/a (Favez et al., 2006), alertando sobre la importancia y el valor del diagnóstico e intervención temprana.

Dado que la calidad y diversidad de las relaciones son fundamentales en la formación del sí mismo, la unidad familiar tríadica ofrece un marco más amplio de relaciones y su evaluación permite abordar más dimensiones del contexto familiar, el desarrollo y la salud mental infantil que las permitidas por las evaluaciones diádicas. El LTP favorece la evaluación de las interacciones familiares desde múltiples perspectivas y desde distintos niveles, tanto desde la evaluación de la alianza familiar, como de posibles desajustes en la interacción que pueden tener consecuencias en el desarrollo psicológico sus miembros.

Junto con esto, el LTP fomenta la incorporación del padre al contexto terapéutico, posibilitando la reflexión de la interacción familiar desde distintas perspectivas. Considerando la importancia de la participación del padre en el desarrollo emocional, cognitivo y social de sus hijos/as, es necesario que los profesionales de la salud incorporen de forma más activa al padre en sus procedimientos estándares de evaluación e intervención. Considerando además que en Chile la mayoría de los padres, de acuerdo a los resultados de la Encuesta IMAGES sí tiene interés en participar junto a sus hijos/as en los diferentes momentos de interacción con el sistema de salud y educativo, se hace necesario impulsar un cambio cultural, en el cual la crianza de los hijos/as sea efectivamente una corresponsabilidad de madres y padres, enfrentando a través de evidencia empírica las creencias tradicionales que consideran como exclusivos de las mujeres los temas de salud, de cuidado o educativos.

Otro aporte relevante del LTP al trabajo de clínicos e investigadores en familia e infancia, está en la contribución que pudiera realizar en familias con padres separados, en donde si bien ya no constituyen un núcleo, continúan formando un equipo en relación a la crianza y las tareas del desarrollo de los hijos/as. El uso del LTP como herramienta clínica en estos casos puede ayudar a los padres a mejorar sus competencias, a visualizar sus dificultades en la coordinación para el adecuado ejercicio de la parentalidad y a reducir el impacto negativo de la separación en los hijos/as.

Resulta importante destacar también, que el material generado a partir de la aplicación del LTP a las familias, constituye una herramienta clínica en sí misma, pudiendo ser utilizado para retroalimentar a los padres a partir de la observación del video, sobre aspectos puntuales de la interacción que se desea favorecer, mejorar o cambiar.

Finalmente se puede concluir que desde que nacen las personas se encuentran inmersas en contextos relacionales familiares, los cuales proveen de una amplia gama de recursos, así como de problemas, que preparan para enfrentar la vida social. Consecuentemente resulta esencial seguir desarrollando investigación en esta área, así como evaluar clínicamente los distintos niveles de funcionamiento familiar, de manera de poder intervenir apropiadamente cuando sea necesario. 


\section{Referencias}

Aguayo, F., Correa, P. \& Cristi, P. (2011). Encuesta IMAGES Chile Resultados de la Encuesta Internacional de Masculinidades y Equidad de Género. Santiago: CulturaSalud/EME.

Allen, S. \& Daly, K. (2007). The effects of father involvement: An updated research summary of the evidence inventory. Guelph, Canada: Centre for Families, Work \& Well-Being, University of Guelph. Recuperado de http://www.fira.ca/cms/documents/29/ Effects_of_Father_Involvement.pdf

Belsky, J. (1981). Early human experience: A family perspective. Developmental Psychology, 17(1), 3-23. doi:10.1037/00121649.17.1.3

Belsky, J., Putnam, S. \& Crnic, K. (1996). Coparenting, parenting, and early emotional development. New directions for child development, 74, 45-55. doi: 10.1002/cd.23219967405

Caldera, Y. \& Lindsey, E. (2006). Coparenting, Mother-Infant Interaction, and Infant-Parent Attachment Relationships in TwoParent Families. Journal of Family Psychology, 20(2), 275-283. doi:10.1037/0893-3200.20.2.275

Centre d'Etude de la Famille. (2007). Figure 2: Views according to the 4 parts of the LTP. Indications for the LTP Setting for Toddlers. Trabajo no publicado, Centro de Estudios de la Familia, Universidad de Lausanna.

Clarke-Stewart, K. (1978). And daddy makes three: The father's impact on mother and young child. Child Development, 49(2), 466-478. doi:10.2307/1128712

Coltrane, S. \& Adams, M. (2001). Men's Family Work: ChildCentered fathering and the Sharing of Domestic Labor. En R. Hertz \& N. Marshall (Eds.), Working Families: The Transformation of the American Home (pp. 72-99). California: University of California Press.

Corboz-Warnery, A. \& Fivaz-Depeursinge, E. (2001). Du couple à la famille: L'alliance prénatale annonce-t-elle le devenir de la famille?. Cahiers critiques de thérapie familiale et de pratique de réseaux, 27, 17-34. doi:10.3917/ctf.027.0017

Cowan, P. \& Cowan, C. (2002). Strengthening couples to improve children's well-being: What we know now. Poverty Research News,6 (3), 18-20. Recuperado de http://www.mdrc.org/publication/policies-strengthen-fatherhood-and-family-relationships

DeBarbieri, T. (1992). Sobre la categoría género. Una introducción teórico-metodológica. Ediciones de las mujeres, 17, 111-128. Recuperado de http://revistas.pucp.edu.pe/index.php/debatesensociologia/article/view/6680/6784

Dornes, M. (2006). Die Seele des Kindes. Entstehung und Entwicklung. Frankfurt: Fischer Taschenbuch Verlag.

Dunn, J. (1991). Young children's understanding of other people: evidence from observations within the family. En D. Frye \& C. Moore (Eds.), Children's Theories of Mind (pp. 97-114). Hillsdale NJ.: Lawrence Erlbaum Associates.

Elliston, D., McHale, J., Talbot, J., Parmley, M. \& Kuersten-Hogan, R. (2008). Withdrawal from coparenting interactions during early infancy. Family Process, 47(4), 481-499. doi:10.1111/j.15455300.2008.00267.x

Fagan, J. \& Iglesias, A. (1999). Father involvement program effects on fathers, father figures, and their Head Start Children: A quasiexperimental study. Early Childhood Research Quartely, 14(2), 243-269. doi:10.1016/S0885-2006(99)00008-3

Favez, N., Frascarolo, F., Carneiro, C., Montfort, V., Corboz-Warnery, A. \& Fivaz-Depeursinge, E. (2006). The development of the family alliance from pregnancy to toddlerhood and children outcomes at 18 months. Infant and Child Development, 15(1), 59-73. doi:10.1002/icd.430.
Feldman, R. (2000). Parents' convergence on sharing and marital satisfaction, father involvement, and parent-child relationship at the transition to parenthood. Infant Mental Health Journal, 21(3), 176-191. doi:10.1002/1097-0355(200007)21:3<176::AIDIMHJ3 $>3.0 . \mathrm{CO} ; 2-4$

Feldman, R. (2007). Parent-infant synchrony and the construction of shared timing; physiological precursors, developmental outcomes, and risk conditions. Journal of Child Psychology and Psychiatry, 48(3-4), 329-354. doi:10.1111/j.1469-7610.2006.01701.x

Fivaz-Depeursinge, E. \& Corboz-Warnery, A. (1999). The primary triangle: A development systems view of mothers, fathers and infants. New York: Basic Books.

Fivaz-Depeursinge, E. \& Favez, N. (2006). Exploring Triangulation in Infancy: Two Contrasted Cases. Family Process, 45(1), 3-18. doi:10.1111/j.1545-5300.2006.00077.x

Fivaz-Depeursinge, E., Frascarolo, F. \& Cornoz-Warnery, A. (1996). Assessing the triadic alliance between fathers, mothers and infants at play. In J. McHale \& P. Cowan (Eds.), Understanding how family-level dynamics affect children's development: studies of two-parent families (pp. 27-44). San Francisco: Jossey-Bass.

Fivaz-Depeursinge, E., Lopes, F., Python, M. \& Favez, N. (2009). Coparenting and Toddler's Interactive Styles in Family Coalitions. Family Process, 48(4), 500-516. doi:10.1111/j.15455300.2009.01298.x

Flykt, M., Kanninen, K., Sinkkonen, J., \& Punamäki, R. (2010). Maternal Depression and Dyadic Interaction: The role of Maternal Attachment Style. Infant and Child Development, 19(5), 530-550. doi: 10.1002/icd.679

Frosch, C. \& Mangelsdorf, S. (2001). Marital behavior, parenting behavior, and multiple reports of preschoolers' behavior problems: mediation or moderation?. Developmental Psychology, 37(4), 502-519. doi:10.1037/0012-1649.37.4.502

Frosch, C., Mangelsdorf, S. \& McHale, J. (2000). Marital behavior and the security of preschooler-parent attachment relationships. Journal of Family Psychology, 14(1), 144-161. doi:10.1037/08933200.14.1.144

Fthenakis, W. (1992). Zur Rolle des Vaters in der Entwicklung des Kindes. Praxis der Psychotherapie und Psychosomatik, 37, 179-189. Recuperado de http://cat.inist.fr/?aModele=afficheN\& cpsidt=5424469

Gere, M., Hagen, K., Villabø, M., Arnberg, K., Neumer, S. \& Torgensen, S. (2013). Fathers' mental health as a protective factor in the relationship between maternal and child depressive symptoms. Depression and Anxiety, 30(1), 31-38. doi:10.1002/da.22010

Gertsch, C., Favez, N., Corboz-Warnery, A. \& Fivaz-Depeursinge, E. (1992). Les débuts de la communication à trois: interactions visuelles triadiques père-mère-bébé. Enfance, 46(4), 323-348. doi:10.3406/enfan.1992.2029

Hedenbro, M. (2006). The family triad-the interaction between the child, its mother, and father from birth to the age of 4 years. Stockholm: Karolinska University Press.

Hofferth, S., Pleck, J., Stueve, J., Bianchi, S. \& Sayer, L. (2002). The demography of fathers: what fathers do. In C. Tamis-LeMonda \& N. Cabrera (Eds.), Handbook of father involvement: interdisciplinary perspectives. Multidisciplinary perspectives (pp. 63-90). New Jersey: Lawrence Erlbaum Associates.

Hyunjeong, S., Young-Joo, P. \& Mi Ja, K. (2006). Predictors of maternal sensitivity during the early postpartum period. Journal of Advanced Nursing, 55(4), 425-434. doi:10.1111/j.13652648.2006.03943.x

Keller, H. (2007). Cultures of Infancy. New Jersey: Lawrence Erlbaum Associates.

Koller, R. (2004). Les affects de l'enfant de trois mois dans l'interaction avec ses deux parents. Switzerland: University of Lausanne, Center for Family Studies. 
Lamb, M. (1977). The Development of Mother-Infant and FatherInfant Attachments in the Second Year of Life. Developmental Psychology, 13(6), 637-648. doi:10.1037/0012-1649.13.6.637

Lamb, M. (1982). Paternal influences on early socio-emotional development. Journal of Child Psychology and Psychiatry, 23(2), 185-190. doi:10.1111/j.1469-7610.1982.tb00063.x

Lamb, M. E., Pleck, J. H., Charnov, E. L. \& Levine, J. A. (1987). A biosocial perspective on paternal behavior and involvement. En J. B. Lancaster, J. Altman, A. Rossi \& R. L. Sherrod (Eds.), Parenting across the life span: Biosocial Dimensions (pp. 11-42). New York: De Gruyter.

Lauretti, A. \& McHale, J. (1997). Shifting patterns of parenting styles between dyadic and family settings: The role of marital quality. Paper presented at the Society for Research in Child Development, Washington, D. C.

Lavanchy, C. (2002). L'interaction visuelle de l'enfant de trois mois avec ses deux parents. Unpublished manuscript. University of Geneva, Switzerland.

McHale, J. (1995). Coparenting and triadic interactions during infancy: The roles of marital distress and child gender. Developmental Psychology, 31(6), 985-996. doi:10.1037/0012-1649.31.6.985

McHale, J. \& Cowan, P. (1996). Editor's Notes. Understanding how family-level dynamics affect children's development: Studies of two-parent families (pp. 1-4). San Francisco: Jossey-Bass.

McHale, J. \& Kuersten-Hogan, R. (2004). Introduction: The dynamics of Raising Children Together. Journal of Adult Development, 11(3), 221-234. doi:10.1023/B:JADE.0000035798.74058.ef

McHale, J., Neugebauer, A., Radin, A. \& Schwartz, A. (1999). Preschoolers' characterizations of multiple family relationships during family doll play. Journal of Clinical Child Psychology, 28(2), 256-268. doi:10.1207/s15374424jccp2802_12

McHale, J. \& Rasmussen, J. (1998). Coparental and family grouplevel dynamics during infancy: Early family precursors of child and family functioning during preschool. Development and Psychopathology, 10(1), 39-59. doi: 10.1017/S0954579498001527

Minuchin, S. (1985). Familias y Terapia Familiar. Barcelona: Gedisa.

Nock, S. \& Einolf, C. (2008). The One Hundred Billion Dollar Man: The Annual Public Costs of Father Absence. Milwaukee: National Fatherhood Initiative.

Olhaberry, M. \& Santelices, M. (2013). Presencia del padre y calidad de la interacción madre-hijo(a): un estudio comparativo en familias monoparentales chilenas nucleares y monoparentales. Universitas Psychologica, 12(3), 833-843. doi:10.11144/Javeriana.UPSY12-3.ppci

Pleck, J. H. (1985). Working Wives/Working Husbands. Beverly Hills: SAGE Publications.

Pleck, E. \& Pleck, J. (1997). Fatherhood ideals in the United States: Historical dimensions. In M. E. Lamb (Ed.), The role of the father in child development (pp. 33-48). New York: John Wiley \& Sons.
Pleck, J. \& Masciadrelli, B. (2004). Paternal involvement by U.S. residential fathers: Levels, sources and consequences. In M. Lamb (Ed.), The Role of the Father in Child Development (pp. 222-271). New Jersey: Wiley.

Rodrigo, M., Martín, J., Cabrera, E. \& Máiquez, M. (2009). Las Competencias Parentales en Contextos de Riesgo Psicosocial. Intervención Psicosocial, 18(2), 113-120. Recuperado de http:// scielo.isciii.es/pdf/inter/v18n2/v18n2a03.pdf

Sarkadi, A., Kristiansson, R., Oberklaid, F. \& Bremberg, S. (2008). Father's involvement and children's developmental outcomes: a systematic review of longitudinal studies. Acta Paediatrica, 97(2), 153-158. doi:10.1111/j.1651-2227.2007.00572.x

Shapiro, A., Gottman, J. \& Carrere, S. (2000). The baby and the marriage: Identifying factors that buffer against decline in marital satisfaction after the first baby arrives. Journal of Family Psychology, 14(1), 59-70. doi:10.1037/0893-3200.14.1.59

Stern, D. (1985). The Interpersonal World of the Infant: A View from Psychoanalysis and Developmental Psychology. New York: Basic Books.

Teti, D., Gelfland, D., Messinger, D. \& Isabella, R. (1995). Maternal Depression and the Quality of Early Attachment: An Examination of Infants, Preschoolers, and Their Mothers. Developmental Psychology, 31(3), 364-376. doi:10.1037/0012-1649.31.3.364

Tronick, E. (1989). Emotions and emotional communication in infants. American Psychologist, 44(2), 112-119. doi:10.1037/0003066X.44.2.112

Valdés, T. \& Olavarría, J. (1998). Masculinidades y equidad de género en América Latina. Santiago: FLACSO-Chile.

Valenzuela, M. (1997). Maternal Sensitivity in a Developing Society: The context of urban poverty and infant chronic undernutrition. Developmental Psychology, 33(5), 845-855. doi:10.1037/00121649.33.5.845

Verschueren, K. \& Marcoen, A. (1999). Representation of self and socioemotional competence in kindergartners: Differential and combined effects of attachment to mother and to father. Child Development, 70(1), 183-201. doi:10.1111/1467-8624.00014

Viziello, G. \& Nosadi, N. (2010). El Lausanne Trialogue Play en la práctica clínica. Revista de Psicopatología y Salud Mental del Niño y del Adolescente, 16, 57-68. Recuperado de http://europa. sim.ucm.es/compludoc/AA?articuloId=793892

Weinberg, M. \& Tronick, E. (1998). The impact of maternal psychiatric illness on infant development. Journal of Clinical Psychiatry, 59(2), 53-61. Recuperado de http://www.ncbi.nlm. nih.gov/pubmed/9559760

Yogman, M. (1981). Games fathers and mothers play with their infants. Infant Mental Health Journal, 2(4), 241-248. doi:10.1002/10970355(198124)2:4<241::AID-IMHJ2280020406>3.0.CO;2-8 